\title{
El uso de antioxidantes: como complemento en el tratamiento de enfermedades cardiovasculares en pacientes con Diabetes Mellitus Tipo II
}

\section{The use of antioxidants: as a complement in the treatment of cardiovascular diseases in patients with type II diabetes mellitus}

Pág. 24-27

Recibido: 22/11/19

Por: Dra. Mariangel Calvo Montero. Aprobado: $30 / 11 / 19$

Licenciada en nutrición humana y dietética, CPN: 1169-12

Máster en Administración de Negocios con Énfasis en Mercadeo

Gerente de la franquicia de suplementos The Vitamin Shoppe

Docente en la Universidad de Ciencias Médicas,San José,Costa Rica

\section{Resumen}

Las complicaciones cardiovasculares son la principal causa de muerte en pacientes diabéticos. El exceso de radicales libres, provocado principalmente por la hiperglicemia que sufren estos pacientes, provoca que los mismos se encuentren en un estado de estrés oxidativo. Esta situación acelera y desarrolla la enfermedad y sus complicaciones. Razón por la cual se estudia la relación y posible efectivo positivo de los antioxidantes para tratar y prevenir la diabetes tipo 2.

Palabras clave: Diabetes Mellitus tipo 2, estrés oxidativo, enfermedades cardiovasculares. vitamina C, antioxidantes, Ácido Alfa Lipoico, vitamina $\mathrm{E}$.

\section{Abstract:}

Cardiovascular complications are the leading cause of death in diabetic patients. The excess of free radicals, caused mainly by the hyperglycemia that these patients suffer, causes them to be in a state of oxidative stress. This situation accelerates and develops the disease and its complications. In this article we are going to study the relationship and possible positive effective of antioxidants to treat and prevent type 2 diabetes.
Keywords: Type 2 diabetes mellitus, oxidative stress, cardiovascular diseases. Vitamin C, antioxidants, Alpha Lipoic Acid, Vitamin E.

Desarrollo:

La Diabetes Mellitus tipo 2 (DM2) es una enfermedad crónica no transmisible de gran preocupación a nivel de salud pública. Es la sétima causa de muerte en Costa Rica y constituye un factor de riesgo para enfermedades cardiovasculares (ECV), que son la primera causa de muerte en el país. (Solis, 2019).

La DM2, también conocida como la diabetes no insulino-dependiente, es la que se presenta con mayor frecuencia. Donde la obesidad es uno de los factores de riesgo mas influyentes. En este tipo de pacientes, se pueden observar secreciones disminuidas de insulina, en respuesta al estímulo fisiológico de la glucosa. Sin embargo, la mayor parte de los pacientes pueden presentar niveles normales e incluso mayores de insulina en respuesta a la glucosa. (Mataix, xxxx).

La obesidad se encuentra relacionada con la DM2 a través de una insulinorresistencia en el músculo esquelético, cardíaco y tejido adiposo. El aumento de los ácidos grasos, en la obesidad, interfiere con la vía glucolítica muscular, afectando la actividad de enzimas. Como por ejemplo la actividad de la fosfofructoquinasa, piruvatoquinasa y la piruvato deshidrigenasa. Además, llega a favorecer la

\section{(ㄷ) $(1) \Theta(9)$}


gluconeogénesis hepática y renal. (Mataix, 2009). Las complicaciones cardiovasculares representan la principal causa de muerte en pacientes con DM2. Esto se da como consecuencia al aumento en las especies reactivas al oxigeno (ROS) y una disminución de las defensas antioxidantes. Los procesos oxidativos están involucrados con la patogénesis, progresión, complicaciones y mal pronóstico de la DM2. El incremento en las concentraciones de glucosa en sangre, han logrado dar a conocer los diversos mecanismos que las ROS inducen en el organismo, ya sea de manera directa o indirecta, y la participación que tienen los agentes oxidantes en estos casos. (Calderón et al. 2013).

Debido a la relación que tiene el aumento del estrés oxidativo con el aumento en las complicaciones cardiovasculares, en pacientes diabéticos. Se llega a la hipótesis de que un mayor consumo de antioxidantes podría ayudar a disminuir el riesgo de desarrollar ECV, en pacientes con diabetes. (Schultz et al,2005).

\section{Antioxidantes}

Los antioxidantes son sustancias que previenen o retrasan la oxidación. Existen dos tipos: Antioxidantes Primarios, que previenen la formación de radicales libres. Como, por ejemplo, las enzimas catalasa y glutatión peroxidasa. Y los Antioxidantes Secundarios, que actúan después de que el radical ya se ha formado. Estos, ceden un electrón provocando que el radical se haga menos reactivo y por lo tanto más fácil de eliminar. En este grupo de antioxidantes se encuentran las vitaminas, bilirrubinas y ácido úrico. (Castellanos de la Cruz et al, 2012).

Un estudio realizado en México con pacientes que padecían DM2 que se les administró el consumo de vitamina $\mathrm{C}$ y $\mathrm{E}$. A los tres meses presentaron niveles de hemoglobina glicosilada y tensión arterial sistólica, considerablemente bajos comparados con el grupo placebo. (Castellanos de la Cruz et al, 2012). Otro estudio realizado indicó que el tratamiento a corto plazco con vitamina $C$ y $E$, reduce la tasa de excreción urinaria de albumina en diabéticos con microalbuminuria (Gaed et al, 2001).

En un estudio de cohorte del European Prospective Investigation of Cancer-Norfolk se les dio seguimiento a 21.831 adultos por un período de 12 años. En dicho estudio se encontró que altos niveles de vitamina $C$ en plasma estaban fuertemente asociados con un bajo riesgo de padecer diabetes (Harding et al, 2008).

Otro antioxidante estudiado en pacientes diabéticos es el ácido alfa lipoico. El mismo actúa como un cofactor esencial de las enzimas respiratorias mitocondriales. Como por ejemplo, del piruvato deshidrogenasa. Además es el cofactor principal para la producción de energía en las mitocondrías. (Ragab et al, 2017).

Mijnhou et al, realizaron una búsqueda de ensayos aleatorizados y controlados sobre el consumo de ácido alfalipoico en pacientes con neuropatía diabética periférica dolorosa. Entre los resultados se puede observar que el consumo de dicho antioxidante disminuye el dolor neuropático de manera significativa y relevante desde el punto de vista clínico.

\section{Conclusiones}

Existe una fuerte relación entre el estrés oxidativo, que sufren los pacientes con diabetes tipo 2 y la progresión de la enfermedad. Varios estudios demuestran como el consumo de antioxidantes puede ayudar, como parte de un tratamiento para evitar la progresión o desarrollo de la enfermedad y sus complicaciones. 


\section{Bibliografía}

Castellanos de la Cruz L, Sanchez Lilliana, Hernandez Nazyeli, et al. Efecto antiglucosilante de las vitaminas C y e en diabéticos versus placebo. (Marzo, 2012). Recuperado de https://www.medigraphic.com/pdfs/ medintmex/mim-2012/mim122d.pdf

Johansen JS, Harris AK, Rychly DJ, Ergul A. Oxidative stress and the use of antioxidants in diabetes: linking basic science to clinical practice. (April, 2005) recuperado de https://www.ncbi.nlm. nih.gov/pmc/articles/PMC1131912/

Ragab A, Samy A, Mostafa T. The Potential Role of a-lipoic acid in Diabetes Mellitus (octubre, 2017). Recuperado de https://www.researchgate.net/ publication/321384471_The_Potential_Role_of_alipoic_acid_in_Diabetes_Mellitus

Mijnhout $\mathrm{G}$, Kollen B, Bilo $\mathrm{H}$, et al Empleo de Ácido Tióctico para el Tratamiento de los Pacientes Diabéticos con Neuropatía Periférica Sintomática: Metanálisis de Estudios Controlados y Aleatorizados. (2012). Recuperado de https://www.siicsalud.com/ pdf/dolor_neuropatico_31918.pdf

Harding $\mathrm{AH}$, Wareham NJ, Bingham SA, et al. Plasma vitamin $C$ level, fruit and vegetable consumption, and the risk of new-onset type 2 diabetes mellitus: the European prospective investigation of cancer-Norfolk prospective study. Arch Intern Med. (julio, 2008). Recuperado de https://www.ncbi.nlm.nih.gov/ pubmed/18663161

Solís María Isabel. Ticos pierden en promedio 13,2 años de vida por diabetes (noviembre, 2019). Recuperado de https://www.ccss.sa.cr/noticia?ticospierden-en-promedio-13-2-anos-de-vida-pordiabetes

Mataix, José. (2009). NUTRICIÓNY ALIMENTACIÓN HUMANA. Barcelona, España. Editorial Oceáno.

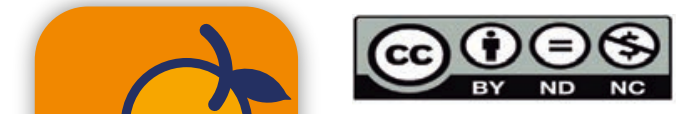




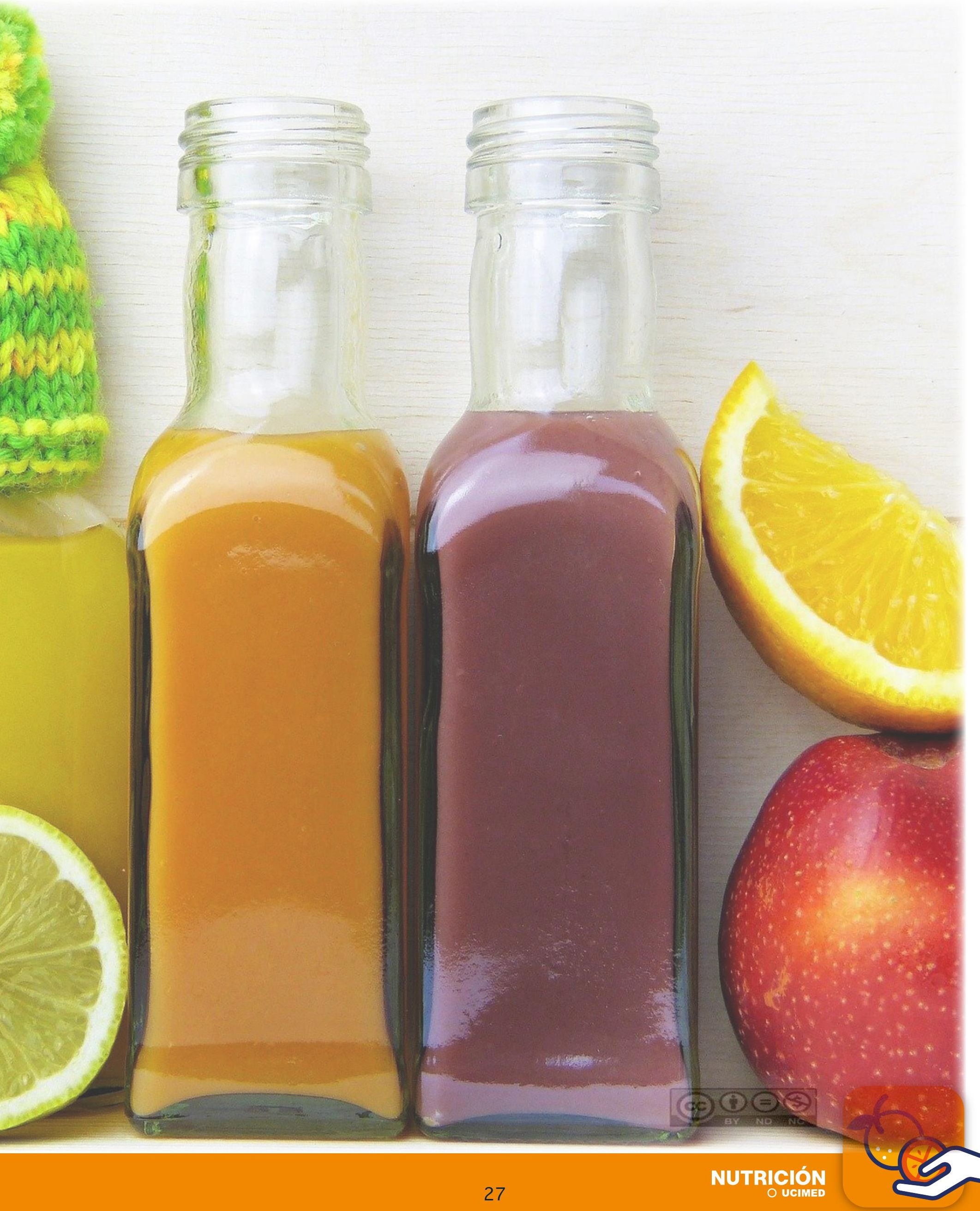

